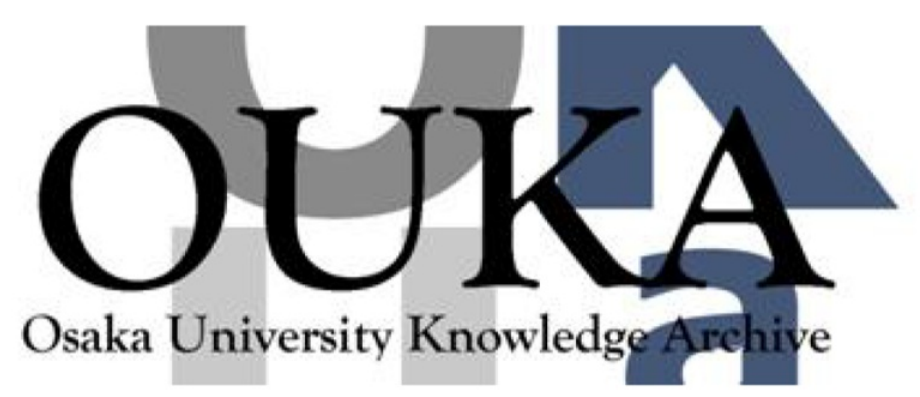

\begin{tabular}{|c|c|}
\hline Title & $\begin{array}{l}\text { Nonlinear behavior of magnetohydrodynamic modes } \\
\text { near marginally stable states. II. Application } \\
\text { to the resistive fast interchange mode }\end{array}$ \\
\hline Author (s) & Nakajima, Noriyoshi; Hamaguchi, Satoshi \\
\hline Citation & Physics of Fluids B. 2(6) p. 1184-p. 1189 \\
\hline Issue Date & $1990-06$ \\
\hline oaire:version & VoR \\
\hline URL & https://hdl. handle. net/11094/78524 \\
\hline rights & $\begin{array}{l}\text { This article may be downloaded for personal use } \\
\text { only. Any other use requires prior permission } \\
\text { of the author and AIP Publishing. This article } \\
\text { appeared in Physics of Fluids B: Plasma Physics } \\
2,1184(1990) \text { and may be found at } \\
\text { https://doi.org/10.1063/1.859254. }\end{array}$ \\
\hline Note & \\
\hline
\end{tabular}

Osaka University Knowledge Archive : OUKA

https://ir. Library. osaka-u. ac. jp/

Osaka University 


\section{Nonlinear behavior of} magnetohydrodynamic modes near marginally stable states. II. Application to the resistive fast interchange mode

Cite as: Physics of Fluids B: Plasma Physics 2, 1184 (1990); https://doi.org/10.1063/1.859254 Submitted: 10 July 1989 . Accepted: 26 February 1990 . Published Online: 01 September 1998

Noriyoshi Nakajima, and Satoshi Hamaguchi 


\title{
Nonlinear behavior of magnetohydrodynamic modes near marginally stable states. II. Application to the resistive fast interchange mode
}

\author{
Noriyoshi Nakajimaa) and Satoshi Hamaguchi \\ Institute for Fusion Studies, The University of Texas at Austin, Austin, Texas 78712
}

(Received 10 July 1989; accepted 26 February 1990)

\begin{abstract}
With the use of the general formulation developed in an earlier paper, the nonlinear evolution of the resistive fast interchange mode near the marginally stable state is obtained analytically. The nonlinear amplitude equation of the mode is shown to be of the Landau type. It is also shown that there is a stable equilibrium bifurcating from the initial equilibrium. Comparing this analytical result to numerical simulations, it is confirmed that the saturation level and the saturation time are well estimated by this Landau type of nonlinear amplitude equation.
\end{abstract}

\section{INTRODUCTION}

In our previous paper, ${ }^{1}$ a general formulation was presented to obtain the nonlinear equation describing time evolution of the mode near the marginally stable state. It was shown there that two different types of nonlinearity exist, depending upon the properties of the linear operator of the mode under consideration-one leads to a Hamiltoniantype equation for the amplitude, and the other leads to a Landau-type equation for the amplitude. ${ }^{2}$ The Hamiltoniantype equation may be obtained in the case where the linear operator is degenerate at the marginally stable state, i.e., when the linear dispersion relation has a double root for the frequency at the marginally stable state. The Landau-type equation may be obtained in the case where the linear operator is nondegenerate, i.e., when the linear dispersion relation has a single root. In magnetohydrodynamics (MHD), the Hamiltonian-type equation may be obtained for nonresonant ideal modes, and the Landau-type equation may be obtained for resistive modes. In Ref. 1, the nonresonant kink mode in reversed field pinches (RFP's) and the quasi-interchange mode in tokamaks were examined by means of this general formulation. ${ }^{1}$ These modes are the nonresonant ideal MHD modes and the corresponding nonlinear amplitude equations are shown to be of the Hamiltonian type. As a result, we find that both the nonresonant kink mode and the quasi-interchange mode are nonlinearly stabilized, and the new stable equilibria bifurcate from the initial equilibrium. This is a manifestation of nonlinear saturation of those modes.

In this paper, we examine the nonlinear behavior of the resistive fast interchange mode, ${ }^{3,4}$ which will be shown to lead to the Landau-type equation of amplitude, a different type from the one obtained for the nonresonant ideal MHD modes in Ref. 1. Although our main goal in this paper is to present an example of the Landau-type equation, it should be noted that the nonlinear evolution of the resistive interchange mode plays an important role in terms of energy confinement in current fusion experiments. ${ }^{5-8}$

The rest of this paper is organized as follows. In Sec. II, we derive the nonlinear amplitude equation for the resistive

\footnotetext{
a) Permanent address: National Institute for Fusion Science, Nagoya 46401, Japan.
}

fast interchange mode, based upon the reduced nonlinear equations used in Ref. 6. Comparison between the analytical results and the numerical simulation results is made in Sec. III, where it is shown that the saturation level and the saturation time are well estimated by the analytical theory. Section IV contains conclusions and discussion.

\section{THE DERIVATION OF THE NONLINEAR AMPLITUDE EQUATION}

We apply the general formulation in Ref. 1 to the resistive fast interchange mode in order to elucidate the saturation mechanism of the mode near the marginally stable state. The resistive interchange mode is an instability caused by the pressure gradient acting against the curvature of the magnetic field in a plasma with finite resistivity. When the resistivity is small and the plasma is stable for ideal interchange modes, which means that Suydam's criterion' is satisfied, i.e., $D<1 / 4$, where the parameter $D$ is specified later, the unstable resistive interchange modes are known to be localized on a particular magnetic surface. ${ }^{3,4}$ There are two different types of resistive interchange mode. One is the resistive slow interchange mode whose solutions must match those of the ideal interchange mode and the other is the resistive fast interchange mode, which has high poloidal and toroidal mode numbers and is localized in the resistive layer. For these localized modes, the original resistive MHD equations describing the modes may be simplified with the use of an asymptotic expansion. Here we only consider the resistive fast interchange mode under the condition that the ideal interchange modes are stable and we use the resistive fast interchange ordering ${ }^{4,5}$ to obtain the dynamical equations of the fluctuations. In the resistive fast interchange ordering, the perturbed density $\rho_{1}$, velocity $\mathbf{v}_{1}$, magnetic field $B_{1}$ and pressure $P_{1}$ are assumed to be of $O(\delta)$, compared to their corresponding equilibrium quantities of $O(1)$, except for $\mathbf{v}_{0}=O\left(\delta^{2}\right)$. Here the subscripts 0 and 1 denote the equilibrium and perturbed quantities, respectively, and $\delta$ is a small parameter of $O(\sqrt{\eta})$ where $\eta$ is the resistivity. It is also assumed that the mode is localized along a mean magnetic field line so that the perpendicular derivative of a fluctuating quantity is of $O\left(\delta^{-1}\right)$ and the parallel derivative of a fluctuating quantity is of $O(1)$. The dynamical equations of the 
resistive fast interchange mode then become, ${ }^{6}$ with the subsidiary expansion in the inverse aspect ratio $\epsilon \sim \beta=2 P_{0} / B_{0}^{2} \ll 1$,

$\frac{\partial \psi}{\partial t}-\nabla_{\|} \varphi-\frac{1}{S} \Delta_{\perp} \psi=-\{\varphi, \psi\}$,

$\frac{\partial}{\partial t} \Delta_{1} \varphi-\nabla_{\|} \Delta_{\perp} \psi+\frac{\partial P}{\partial y}-M \Delta_{l}^{2} \varphi=-\left\{\varphi, \Delta_{\perp} \varphi\right\}+\left\{\psi, \Delta_{1} \psi\right\}$,

$\frac{\partial P}{\partial t}+D \frac{\partial \varphi}{\partial y}-\chi \Delta_{1} P=-\{\varphi, P\}$

The dependent variables in Eqs. (1)-(3) are defined by

$$
\psi \equiv \frac{1}{r_{s} B_{\theta 0} B_{0}} \psi_{1}, \quad \varphi \equiv \frac{\sqrt{\rho_{0}}}{r_{s} B_{\theta 0} B_{0}} \varphi_{1},
$$

and

$$
P \equiv\left(2 / B_{0}^{2}\left|\sigma_{0}\right|^{3 / 2}\right) P_{1},
$$

and the independent variables are defined by

$$
x \equiv\left|\sigma_{0}\right|^{1 / 2} \frac{r-r_{s}}{r_{s}}, \quad y \equiv \frac{B_{\theta 0}\left|\sigma_{0}\right|^{1 / 2}}{r_{s} B_{0}}\left(z-\mu_{0} \theta\right),
$$

and

$$
t \equiv\left(B_{\theta 0}\left|\sigma_{0}\right| / r_{s} \sqrt{\rho_{0}}\right) \tau .
$$

Here, $(r, \theta, z)$ denote the polar coordinates of the cylinder in which the plasma is contained; $\tau$ denotes the time; $r=r_{s}$ is the radius of the resonant surface; and $B_{0}, B_{\theta 0}$, and $B_{20}$ indicate the absolute value and the azimuthal and longitudinal components of the equilibrium magnetic field $\mathbf{B}_{0}$, respectively. The perturbed magnetic field $B_{1}$ and the perturbed velocity field $\nabla_{1}$ are expressed by $B_{1}=\nabla_{\perp} \psi_{1} \times \mathbf{b}$ and $\nabla_{1}=\nabla_{1} \varphi_{1} \times b$, where $b=B_{0} / B_{0}^{2}$. The parameters used here are defined by

$$
\begin{aligned}
& D \equiv-2 r_{s} P_{0}^{\prime} / B_{0}^{2} \sigma_{0}^{2}, \quad S \equiv r_{s} B_{\theta 0} / \eta \sqrt{\rho_{0}}, \\
& M \equiv \mu_{1} / r_{s} B_{\theta 0} \sqrt{\rho_{0}}, \quad \chi \equiv(\Gamma-1) \kappa_{1} / r_{s} B_{\theta 0} \sqrt{\rho_{0}}, \\
& \mu_{0} \equiv r_{s} B_{20} / B_{\theta 0}, \quad \sigma_{0} \equiv B_{\theta 0} \mu_{0}^{\prime} / B_{0} .
\end{aligned}
$$

In Eqs. (6) a prime denotes $d / d r, \Gamma$ is the ratio of the specific heats, and $\mu_{1}$ and $\kappa_{1}$ indicate the perpendicular viscosity and the perpendicular heat conductivity, respectively. Note that all the equilibrium quantities are evaluated at the mode rational surface $r=r_{s}$. The parallel diffusion coefficients are ignored for simplicity. In the following argument, we only consider nonlinear saturation of the mode occurring near the marginally stable state with small amplitude. Therefore, we may ignore nonlinear interactions of the modes with different helicities localized on different resonant surfaces and we only need to take into account nonlinear interaction of the single helicity modes, the helicity of which is given by $\mu_{0}$. Therefore, the parallel derivatives become $\nabla_{\|}=x \partial / \partial y$ in these equations. The Poisson bracket $\{$,$\} and the perpendic-$ ular Laplacian are defined by

$$
\{u, v\} \equiv \frac{\partial u}{\partial x} \frac{\partial v}{\partial y}-\frac{\partial u}{\partial y} \frac{\partial v}{\partial x}, \quad \Delta_{1} u \equiv \frac{\partial^{2} u}{\partial x^{2}}+\frac{\partial^{2} u}{\partial y^{2}} .
$$

The domain of the mode is defined as $|x| \leqslant \delta_{x}$ and $|y| \leqslant \delta_{y}$ with $\delta_{x}$ and $\delta_{y}$ being positive constants of order $S^{-1 / 2}$, which represents the narrow boundary layer within which the mode is considered to be localized. As in the case of the linear resistive fast interchange mode, ${ }^{4}$ we assume that the mode decays rapidly away from its mode rational surface $x=0$ and $\psi, \varphi$, and $P$ are periodic in $y$ with period $2 \delta_{y}$. Here we note that the plasma is assumed to be stable for ideal MHD modes and that the mode under consideration is restricted to the resistive fast interchange mode so that the localization of the resistive mode along the mode rational surface is justified.

We now consider the situation where the equilibrium pressure gradient, indicated by the parameter $D$, is slightly larger than its critical value $D_{c}$ (which corresponds to the marginally stable state and will be determined later as an eigenvalue of the linearized system) but satisfies Suydam's criterion, i.e., $D_{c} \leqslant D<1 / 4$, so that only the resistive interchange mode is weakly linear unstable. It should be noted that the resistive fast interchange mode near the marginally stable state has a similar eigenfunction to the marginally stable state, ${ }^{6}$ so that the following expansion around the marginally stable state is valid. It is known that this unstable mode has no real frequency and that the functions $\psi, \varphi$, and $P$ have odd, even, and even symmetry with respect to $x$, respectively. ${ }^{6}$

We apply the general formulation in Ref. 1 to Eqs. (1)(3) as follows. The expanded forms of $\psi, \varphi$, and $P$ are given by

$$
\left[\begin{array}{l}
\psi \\
\varphi \\
P
\end{array}\right]=\lambda\left[\begin{array}{l}
\psi_{1} \\
\varphi_{1} \\
P_{1}
\end{array}\right]+\lambda^{2}\left[\begin{array}{l}
\psi_{2} \\
\varphi_{2} \\
P_{2}
\end{array}\right]+\lambda^{3}\left[\begin{array}{l}
\psi_{3} \\
\varphi_{3} \\
P_{3}
\end{array}\right]+\cdots,
$$

where $\lambda$ is an ordering parameter. We introduce the following form for $\psi_{1}$ and also the multiple-time-scale method:

$$
\begin{aligned}
& \psi_{1}=A \psi_{1}(x) \cos k y, \\
& \tau_{1}=\lambda t, \quad \tau_{2}=\lambda^{2} t, \ldots, \\
& \frac{\partial}{\partial t}=\lambda \frac{\partial}{\partial \tau_{1}}+\lambda^{2} \frac{\partial}{\partial \tau_{2}}+\cdots, \\
& A=A\left(\tau_{1}, \tau_{2}, \ldots\right),
\end{aligned}
$$

where $A$ and $\psi_{1}(x)$ are real functions. In Eq. (11), we make use of the fact that the real frequency vanishes. Finally, we choose the mean pressure gradient $D$ to be the parameter $p$ in the general formulation, so that

$$
D=D_{c} \pm \lambda^{2} \text {, }
$$

where $D_{c}$ denotes the critical value (or the linear stability limit) of $D$ and the plus sign corresponds to the linearly unstable situation. Substituting Eqs. (8)-(13) into Eqs. (1) - (3) yields simultaneous equations for each order of $\lambda$, which we solve beginning from the lowest order.

\section{A. Order $\lambda$}

This order corresponds to the marginally stable state, and we have the following linearized equations: 
$L\left[\begin{array}{l}\varphi_{1} \\ P_{1}\end{array}\right]=\left[\begin{array}{cc}-S x^{2} \frac{\partial^{2} \varphi_{1}}{\partial y^{2}}+M \Delta_{1}^{2} \varphi_{1} & -\frac{\partial P_{1}}{\partial y} \\ \frac{\partial \varphi_{1}}{\partial y} & -\frac{\chi}{D_{c}} \Delta_{1} P_{1}\end{array}\right]=0$

and

$$
\Delta_{1} \psi_{1}=-S x \frac{\partial \varphi_{1}}{\partial y} .
$$

Assuming $\psi_{1}$ is given by Eq. (9), we may have the following type of linear solutions:

$$
\left[\begin{array}{l}
\psi_{1} \\
\varphi_{1} \\
P_{1}
\end{array}\right]=A\left[\begin{array}{l}
\psi_{1}(x) \cos k y \\
\varphi_{1}(x) \sin k y \\
P_{1}(x) \cos k y
\end{array}\right]
$$

The real functions $\psi_{1}(x), \varphi_{1}(x), P_{1}(x)$, and $D_{c}$ are, respectively, the eigenfunctions and the eigenvalue of the following eigenvalue problem:

$$
L_{1}\left[\begin{array}{c}
\varphi_{1}(x) \\
P_{1}(x)
\end{array}\right]=0
$$

where $\varphi_{1}(x)=P_{1}(x)=\varphi_{1}^{\prime}(x)=0$ at $|x|=\delta_{x}$, and

$$
\left(\partial_{x}^{2}-k^{2}\right) \psi_{1}(x)=-S k x \varphi_{1}(x)
$$

with $\psi_{1}(x)=0$ at $|x|=\delta_{x}$. Here the linear operator $L_{1}$ is given by the $l=1$ case of the following operator:

$L_{l} \equiv\left[\begin{array}{cc}S(l k x)^{2}+M\left[\partial_{x}^{2}-(l k)^{2}\right]^{2} & l k \\ l k & -\frac{\chi}{D_{c}}\left[\partial_{x}^{2}-(l k)^{2}\right]\end{array}\right]$

It is seen that $L_{l}$ is a Hermitian operator with respect to the following inner product:

$$
\left(u_{1}, u_{2}\right)\left[\begin{array}{l}
v_{1} \\
v_{2}
\end{array}\right]=\left\langle u_{1} v_{1}+u_{2} v_{2}\right\rangle
$$

where

$$
\langle w\rangle \equiv \frac{1}{2 \delta_{x}} \int_{-\delta_{x}}^{\delta_{x}} d x^{\prime} w\left(x^{\prime}\right) .
$$

We assume in what follows that the solutions of Eqs. (17) and (18) are unique.

\section{B. Order $\lambda^{2}$}

The equations in this order are given by

$$
L\left[\begin{array}{c}
\varphi_{2} \\
P_{2}
\end{array}\right]=\left[\begin{array}{c}
\left\{\varphi_{1}, \Delta_{1} \varphi_{1}\right\}-S x \frac{\partial}{\partial y}\left\{\varphi_{1}, \psi_{1}\right\}-\left\{\psi_{1}, \Delta_{1} \psi_{1}\right\}+\frac{\partial}{\partial \tau_{1}} \Delta_{1} \varphi_{1}-S x \frac{\partial^{2}}{\partial \tau_{1} \partial y} \psi_{1} \\
-\frac{1}{D_{c}}\left(\left\{\varphi_{1}, P_{1}\right\}+\frac{\partial}{\partial \tau_{1}} P_{1}\right)
\end{array}\right]
$$

and

$$
\Delta_{1} \psi_{2}=-S x \frac{\partial \varphi_{2}}{\partial y}+S\left\{\varphi_{1}, \psi_{1}\right\}+S \frac{\partial \psi_{1}}{\partial \tau_{1}} .
$$

From the inhomogeneous terms of Eqs. (22) and (23), we see that $\psi_{2}, \varphi_{2}$ and $\boldsymbol{P}_{2}$ have components with the same phase in $y$ as the order- $\lambda$ solutions: $\psi_{21}(x) \cos k y, \varphi_{21}(x) \sin k y$, and $P_{21}(x) \cos k y$. Here the functions $\psi_{21}(x), \varphi_{21}(x)$, and $P_{21}(x)$ are given by

$$
\begin{aligned}
& L_{1}\left[\begin{array}{l}
\varphi_{21}(x) \\
P_{21}(x)
\end{array}\right] \\
& \quad=\frac{\partial A}{\partial \tau_{1}}\left[\begin{array}{c}
\left(\partial_{x}^{2}-k^{2}\right) \varphi_{1}(x)+S k x \psi_{1}(x) \\
-\frac{1}{D_{c}} P_{1}(x)
\end{array}\right],
\end{aligned}
$$

with $\varphi_{21}(x)=P_{21}(x)=\varphi_{21}^{\prime}(x)=0$ at $|x|=\delta_{x}$, and

$$
\left(\partial_{x}^{2}-k^{2}\right) \psi_{21}(x)=-S k x \varphi_{21}(x)+\frac{\partial A}{\partial \tau_{1}} S \psi_{1}(x),
$$

with $\psi_{21}(x)=0$ at $|x|=\delta_{x}$. From the solvability condition of Eq. (24), we obtain

$$
\begin{aligned}
& \frac{\partial A}{\partial \tau_{1}}\left\langle-\left\{\left[\partial_{x} \varphi_{1}(x)\right]^{2}+\left[k \varphi_{1}(x)\right]^{2}\right\}+\left\{\left[\partial_{x} \psi_{1}(x)\right]^{2}\right.\right. \\
& \left.\left.+\left[k \psi_{1}(x)\right]^{2}\right\}-\frac{1}{D_{c}} P_{1}^{2}(x)\right)=0 .
\end{aligned}
$$

The averaged quantity $(\cdots)$ in Eq. (26) does not vanish generally, and hence we have

$$
\frac{\partial A}{\partial \tau_{1}}=0
$$

Then, the inhomogeneous terms of Eqs. (24) and (25) vanish, and so we have the solutions proportional to the order- $\lambda$ solutions. According to the general formulation, however, these solutions could be transferred into the order- $\lambda$ ones by redefinition of the coefficient $A$, and therefore we set $\psi_{21}(x)=\varphi_{21}(x)=P_{21}(x)=0$. Then, from Eqs. (22) and (23) we have

$$
\left[\begin{array}{l}
\psi_{2} \\
\varphi_{2} \\
P_{2}
\end{array}\right]=A^{2}\left[\begin{array}{l}
\psi_{20}(x) \\
\varphi_{20}(x) \\
P_{20}(x)
\end{array}\right]+A^{2}\left[\begin{array}{l}
\psi_{22}(x) \cos 2 k y \\
\varphi_{22}(x) \sin 2 k y \\
P_{22}(x) \cos 2 k y
\end{array}\right],
$$

where $\psi_{2 j}(x), \varphi_{2 j}(x)$, and $P_{2 j}(x)(j=0,2)$ may be obtained from the following equations: 


$$
L_{0}\left[\begin{array}{l}
\varphi_{20}(x) \\
P_{20}(x)
\end{array}\right]=\left[\begin{array}{c}
0 \\
\frac{k}{2 D_{c}} \partial_{x}\left[\varphi_{1}(x) P_{1}(x)\right]
\end{array}\right]
$$

with $\varphi_{20}(x)=P_{20}(x)=\varphi_{20}^{\prime}(x)=0$ at $|x|=\delta_{x}$,

$$
\partial_{x}^{2} \psi_{20}(x)=-(k S / 2) \partial_{x}\left[\varphi_{1}(x) \psi_{1}(x)\right],
$$

with $\psi_{20}(x)=0$ at $|x|=\delta_{x}$, and

$L_{2}\left[\begin{array}{c}\varphi_{22}(x) \\ P_{22}(x)\end{array}\right]=\left[\begin{array}{c}\frac{k f}{2} \\ -\frac{k}{2 D_{c}} P_{1}^{2}(x) \partial_{x}\left(\frac{\varphi_{1}(x)}{P_{1}(x)}\right)\end{array}\right]$,

with $\varphi_{22}(x)=P_{22}(x)=\varphi_{22}^{\prime}(x)=0$ at $|x|=\delta_{x}$,

$$
\begin{aligned}
& {\left[\partial_{x}^{2}-(2 k)^{2}\right] \psi_{22}(x)} \\
& \quad=-2 S k x \varphi_{22}(x)+\frac{k S}{2} \psi_{1}^{2}(x) \partial_{x}\left(\frac{\varphi_{1}(x)}{\psi_{1}(x)}\right)
\end{aligned}
$$

with $\psi_{22}(x)=0$ at $|x|=\delta_{x}$, where

$$
\begin{aligned}
f \equiv & -\varphi_{1}^{2}(x) \partial_{x}\left(\frac{\left(\partial_{x}^{2}-k^{2}\right) \varphi_{1}(x)}{\varphi_{1}(x)}\right) \\
& -\psi_{1}^{2}(x) \partial_{x}\left(\frac{\left(\partial_{x}^{2}-k^{2}\right) \psi_{1}(x)}{\psi_{1}(x)}\right) \\
& +2 S x \psi_{1}^{2}(x) \partial_{x}\left(\frac{\varphi_{1}(x)}{\psi_{1}(x)}\right) .
\end{aligned}
$$

Here we note that $\varphi_{20}(x)=0$.

\section{Order $\lambda^{3}$}

In order to obtain the nonlinear equation for $A$, we consider the solutions $\psi_{31}, \varphi_{31}$, and $P_{31}$ that have the same phase in $y$ as that of the order- $\lambda$ solutions, i.e., $\psi_{31}(x) \cos k y$, $\varphi_{31}(x) \sin k y$, and $P_{31}(x) \cos k y$. The functions $\psi_{31}(x)$, $\varphi_{31}(x)$, and $P_{31}(x)$ are the solutions of the following equations:

$$
L_{1}\left[\begin{array}{c}
\varphi_{31}(x) \\
P_{31}(x)
\end{array}\right]=\left[\begin{array}{l}
A^{3} f_{1}+\frac{\partial A}{\partial \tau_{2}}\left[\left(\partial_{x}^{2}-k^{2}\right) \varphi_{1}(x)+S k x \psi_{1}(x)\right] \\
-A^{3} \frac{g_{1}}{D_{c}}-( \pm 1) A \frac{k}{D_{c}} \varphi_{1}(x)-\frac{\partial A}{\partial \tau_{2}} \frac{1}{D_{c}} P_{1}(x)
\end{array}\right],
$$

with $\varphi_{31}(x)=P_{31}(x)=\varphi_{31}^{\prime}(x)=0$ at $|x|=\delta_{x}$, and

$$
\left(\partial_{x}^{2}-k^{2}\right) \psi_{31}(x)=-S k x \varphi_{31}(x)+A^{3} S h_{1}+\frac{\partial A}{\partial \tau_{2}} S \psi_{1}(x),
$$

with $\psi_{31}(x)=0$ at $|x|=\delta_{x}$, where

$$
\begin{aligned}
f_{1} \equiv & k\left\{-\partial_{x} \varphi_{1}(x)\left[\partial_{x}^{2}-(2 k)^{2}\right] \varphi_{22}(x)+\varphi_{22}(x) \partial_{x}\left(\partial_{x}^{2}-k^{2}\right) \varphi_{1}(x)-\frac{1}{2} \varphi_{1}(x) \partial_{x}\left[\partial_{x}^{2}-(2 k)^{2}\right] \varphi_{22}(x)\right. \\
& +\frac{1}{2} \partial_{x} \varphi_{22}(x)\left(\partial_{x}^{2}-k^{2}\right) \varphi_{1}(x)+\partial_{x} \psi_{1}(x)\left[\partial_{x}^{2}-(2 k)^{2}\right] \psi_{22}(x)-\psi_{22}(x) \partial_{x}\left(\partial_{x}^{2}-k^{2}\right) \psi_{1}(x) \\
& +\frac{1}{2} \psi_{1}(x) \partial_{x}\left[\partial_{x}^{2}-(2 k)^{2}\right] \psi_{22}(x)-\frac{1}{2} \partial_{x} \psi_{22}(x)\left(\partial_{x}^{2}-k^{2}\right) \psi_{1}(x) \\
& \left.-\psi_{1}(x) \partial_{x}^{3} \psi_{20}(x)+\partial_{x} \psi_{20}(x)\left(\partial_{x}^{2}-k^{2}\right) \psi_{1}(x)+S x h_{1}\right\} \\
g_{1} \equiv & -k\left[\varphi_{1}(x) \partial_{x} P_{20}(x)+\partial_{x} \varphi_{1}(x) P_{22}(x)+\varphi_{22}(x) \partial_{x} P_{1}(x)+\frac{1}{2} \varphi_{1}(x) \partial_{x} P_{22}(x)+\frac{1}{2} \partial_{x} \varphi_{22}(x) P_{1}(x)\right] \\
h_{1} \equiv & -k\left[\varphi_{1}(x) \partial_{x} \psi_{20}(x)+\partial_{x} \varphi_{1}(x) \psi_{22}(x)+\varphi_{22}(x) \partial_{x} \psi_{1}(x)+\frac{1}{2} \varphi_{1}(x) \partial_{x} \psi_{22}(x)+\frac{1}{2} \partial_{x} \varphi_{22}(x) \psi_{1}(x)\right]
\end{aligned}
$$

In Eq. (34), the term involving the factor of \pm 1 comes from Eq. (13). The solvability condition of Eq. (34) yields the following nonlinear equation, as was proved in the general formulation ${ }^{1}$ in the nondegenerate case:

$$
d_{0} \frac{\partial A}{\partial \tau_{2}} \pm d_{1} A+d_{3} A^{3}=0
$$

where

$$
\begin{aligned}
d_{0} \equiv & \left\langle-\left\{\left[\partial_{x} \varphi_{1}(x)\right]^{2}+\left[k \varphi_{1}(x)\right]^{2}\right\}\right. \\
& \left.+\left\{\left[\partial_{x} \psi_{1}(x)\right]^{2}+\left[k \psi_{1}(x)\right]^{2}\right\}-\left(1 / D_{c}\right) P_{1}^{2}(x)\right\rangle,
\end{aligned}
$$

$d_{1} \equiv\left(\chi / D_{c}^{2}\right)\left\langle\left[\partial_{x} P_{1}(x)\right]^{2}+\left[k P_{1}(x)\right]^{2}\right\rangle$, $d_{3} \equiv\left\langle\varphi_{1}(x) f_{1}-\left(1 / D_{c}\right) P_{1}(x) g_{1}\right\rangle$.

Rewriting Eq. (39) in terms of the original variables, i.e., $t=\tau_{2} / \lambda^{2}$ and $\mathscr{A}=\lambda A$, we have the nonlinear amplitude equation:

$$
d_{0} \frac{d \mathscr{A}}{d t}+\left(D-D_{c}\right) d_{1} \mathscr{A}+d_{3} \mathscr{A}^{3}=0,
$$

where we used Eq. (13). Defining

$$
\begin{aligned}
& \sigma \equiv-\left(D-D_{c}\right) d_{1} / d_{0}, \\
& l \equiv 2 d_{3} / d_{0},
\end{aligned}
$$




$$
\frac{d \mathscr{A}^{2}}{d t}=2 \sigma \mathscr{A}^{2}-l \mathscr{A}^{4}
$$

This equation is the Landau-type equation well known in fluid dynamics, ${ }^{2}$ the solution of which is expressed analytically as

$$
\mathscr{A}^{2}=\frac{\mathscr{A}_{0}^{2}}{(l / 2 \sigma) \mathscr{A}_{0}^{2}+\left[1-(l / 2 \sigma) \mathscr{A}_{0}^{2}\right] e^{-2 \sigma t}},
$$

where $\mathscr{A}_{0}$ is the initial amplitude. It should be noted that $\sigma$ gives the linear growth rate of the mode.

The solution, Eq. (47), exhibits wide classes of nonlinear phenomena, depending upon the signs of the coefficients $\sigma$ and $l$. For example, consider the linearly unstable case, i.e., $\sigma>0$. In this case, if the mode under consideration cannot be nonlinearly stabilized by the second term on the right-hand side of Eq. (46), i.e., $l<0$, the solution becomes unbounded in time, so that none of the higher-order terms may be truncated and there is a fast transition to turbulence. On the other hand, if the mode is nonlinearly stabilized, i.e., $l>0$, then a new stable equilibrium bifurcating from the initial equilibrium is obtained. In this case, the amplitude $\mathscr{A}$ asymptotically approaches its saturation level $\mathscr{A}_{e}$ given by

$$
\mathscr{A}_{e}=\sqrt{2 \sigma / l}=\sqrt{-\left(D-D_{c}\right) d_{1} / d_{3}},
$$

for any positive initial amplitude $\mathscr{A}_{0}>0$.

It is easy to show that this saturation amplitude $\mathscr{A}_{e}$ is identical to the one given in Ref. 6, which was obtained by a somewhat different nonlinear analysis. In the perturbation method presented in this paper, however, we also obtain a nonlinear time evolution of the mode from the initial amplitude $\mathscr{A}_{0}$, which was not assessed in Ref. 6. Such time evolution of the mode at the initial stage is characterized by the saturation time $\Delta \tau$, or the time that elapses from $\mathscr{A}=0.1 \mathscr{A}_{e}$ to $\mathscr{A}=0 . \mathscr{A}_{e}$, which is given by

$$
\Delta \tau \simeq 3 / \sigma \text {. }
$$

\section{COMPARISON BETWEEN THEORY AND NUMERICAL SIMULATIONS}

In this section, we present sample calculations of the nonlinear evolution of the resistive fast interchange mode, using both theory [Eq. (46)] and direct numerical simulations of the nonlinear system of Eqs. (1)-(3). The coefficients $\sigma$ and $l$ in Eq. (46), given by Eqs. (44) and (45), respectively, are calculated from the solutions of the linear equations (17), (18) and (29)-(32). The details of the numerical simulations of Eqs. (1)-(3) may be found in Ref. 6 . Following Ref. 6, we use the scale transformation

$$
\begin{aligned}
& x \rightarrow S^{-1 / 2} x, \quad y \rightarrow S^{-1 / 2} y, \\
& \psi \rightarrow S^{-1} \psi, \quad \varphi \rightarrow S^{-1} \varphi, \quad P \rightarrow S^{-1 / 2} P,
\end{aligned}
$$

and introduce new parameters $M_{s}=S M$ and $\chi_{s}=S \chi$ in order to eliminate the explicit dependence of the system on the parameter $S$. The following values were chosen for parameters in our calculations:

$$
\begin{aligned}
& M_{s}=1.0, \quad \chi_{s}=0.1, \\
& \delta_{x} S^{1 / 2}=25, \quad \delta_{y} S^{1 / 2}=\pi .
\end{aligned}
$$

In the numerical simulations of Eqs. (1)-(3), 150 grid points in the $x$ direction and seven Fourier modes $(0 \leqslant m \leqslant 6)$ in the $y$ direction were employed, where the mode number $m$ is defined by the relation $\partial / \partial y=k=m \pi / \delta_{y}$. It is confirmed that these grid points and the number of Fourier modes give sufficient numerical resolution to obtain correct mode saturation in the calculation presented in this section. With these parameters, we obtain the eigenvalue $D_{c}$ from Eq. (17),

$$
D_{c}=0.085 \text {, }
$$

and the parameters of Eqs. (44) and (45),

$$
\sigma /\left(D-D_{c}\right)=3.8 \times 10^{-1}, \quad l=1.2 \times 10^{5} \text {. }
$$

Because $l \gg \sigma /\left(D-D_{c}\right)$, the mode under consideration is nonlinearly stabilized when $D \gtrsim D_{c}$.

Figure 1 shows time evolution of the normalized energy $E$ for the case of $D=0.13\left(>D_{c}\right)$ as calculated from the theory [Eq. (46)] (solid curve) and as obtained from numerical simulation of the system of Eqs. (1)-(3) (broken curve), where

$$
E=\frac{1}{2 \delta_{y} \Delta} \int_{-\delta_{x}}^{\delta_{x}} d x \int_{-\delta_{y}}^{\delta_{y}} d y\left[\left(\frac{\partial \varphi}{\partial x}\right)^{2}+\left(\frac{\partial \varphi}{\partial y}\right)^{2}\right]
$$

with the typical mode width $\Delta$ in the $x$ direction given by $\Delta=\delta_{y}{ }^{6}$ The analytical result is in good agreement with the simulation result. The overshooting that appears in the numerical simulation seems to manifest a higher order nonlinear correction, which is not included in Eq. (46).

\section{CONCLUSIONS AND DISCUSSION}

Using the recently developed general formulation, ${ }^{1}$ we have examined the nonlinear evolution of the resistive fast interchange mode analytically. In the case of the resistive fast interchange mode, the linear operator is nondegenerate at the marginally stable state, i.e., the linear dispersion relation of the mode has a single root of the frequency at the marginally stable state. Therefore, as shown in Ref. 1, the nonlinear amplitude equation of this mode turns out to be of the Landau type. It is found that a new stable equilibrium bifurcates from the initial equilibrium. Comparison between the perturbation theory and numerical simulations of the

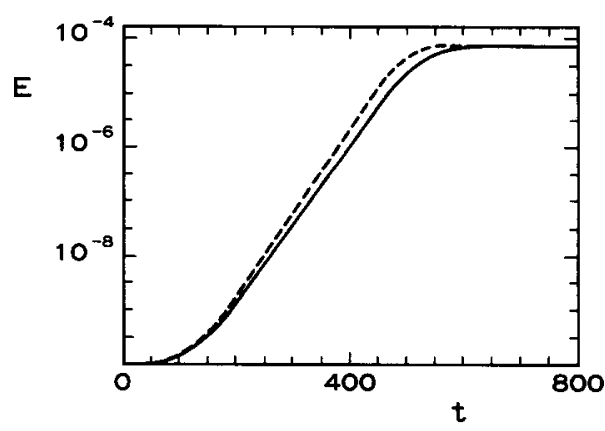

FIG. 1. Time evolution of the normalized energy $E$ in Eq. (54) for $D=0.13$. Analytical and numerical results are shown by a solid curve and a broken curve, respectively. The unit of the time $t$ is defined in Eq. (5). 
system of Eqs. (1)-(3) was made, from which we confirm that the saturation level and the saturation time are well estimated from this nonlinear amplitude equation.

\section{ACKNOWLEDGMENTS}

The authors wish to express their thanks to Professor $H$. Berk, Professor R. Hazeltine, and Professor W. Horton for their fruitful discussions and encouragement. We are also grateful to Dr. J. Van Dam for suggesting several improvements in the manuscript.

This work was supported in part by the U.S. Department of Energy Contract No. DE-FG05-80ET-53088. Part of this work was done while one of the authors (N.N.) was a
Visiting Scientist under the auspices of the U.S.-Japan Joint Institute for Fusion Theory.

'N. Nakajima, Phys. Fluids B 2, 1170 (1990).

${ }^{2}$ P. G. Drazin and W. H. Reid, Hydrodynamic Stability (Cambridge U.P., Cambridge, 1981).

${ }^{3}$ H. P. Furth, J. Killeen, and M. N. Rosenbluth, Phys. Fluids 6, 459 (1963). ${ }^{4}$ B. Coppi, J. M. Greene, and J. L. Johnson, Nucl. Fusion 6, 101 (1966).

${ }^{5}$ A. Bhattacharjee and E. Hameiri, Phys. Rev. Lett. 57, 206 (1986).

'S. Hamaguchi, Phys. Fluids B 1, 1416 (1989).

${ }^{7}$ J. W. Connor and J. B. Taylor, Phys. Fluids 27, 2676 (1984).

${ }^{8}$ M. Yagi, M. Wakatani, and A. Hasegawa, J. Phys. Soc. Jpn. 56, 973 (1987).

${ }^{9}$ B. R. Suydam, in Proceedings of the Second International Conference on Peaceful Uses of Atomic Energy (United Nations, Geneva, 1958), Vol. 31, p. 157. 